\title{
Cardiac tumours: non-invasive detection and assessment by gated cardiac blood pool radionuclide imaging
}

\author{
DAVID PITCHER, RAY WAINWRIGHT, DAVID BRENNAND-ROPER, \\ PHILIP DEVERALL, EDGAR SOWTON, MICHAEL MAISEY
}

From the Departments of Cardiology, Cardiothoracic Surgery and Nuclear Medicine, Guy's Hospital, London

SUMMARY Four patients with cardiac tumours were investigated by gated cardiac blood pool radionuclide imaging and echocardiography. Contrast angiocardiography was performed in three of the cases. Two left atrial tumours were detected by all three techniques. In one of these cases echocardiography alone showed additional mitral valve stenosis, but isotope imaging indicated tumour size more accurately. A large septal mass was detected by all three methods. In this patient echocardiography showed evidence of left ventricular outflow obstruction, confirmed at cardiac catheterisation, but gated isotope imaging provided a more detailed assessment of the abnormal cardiac anatomy. In the fourth case gated isotope imaging detected a large right ventricular tumour which had not been identified by echocardiography.

Gated cardiac blood pool isotope imaging is a complementary technique to echocardiography for the non-invasive detection and assessment of cardiac tumours.

Cardiac tumours are rare lesions. Formerly contrast angiography was the only available method to establish the diagnosis. Echocardiography is a sensitive non-invasive technique for the detection of cardiac tumours, particularly in the left atrium ${ }^{12}$ and gated cardiac blood pool radionuclide imaging has been used as an additional atraumatic method to detect left atrial myxomata. ${ }^{3} 4$

Over a six-month period we have investigated four patients with intracardiac tumours, using gated cardiac blood pool imaging in addition to other techniques. In this report we describe the appearance of these tumours.

\section{Imaging methods}

Gated cardiac blood pool images were acquired in each case using an Ohio Nuclear Series 100 scintillation camera and a high resolution, parallelhole collimator, interfaced to a dedicated minicomputer (Digital Equipment Corporation, Gamma 11). Twenty $\mathrm{mCi}$ of technetium- $99 \mathrm{~m}$ labelled autologous red blood cells were injected intravenously and after equilibration of the tracer within the blood pool the camera was positioned over the Received for publication 5 December 1979 patient's praecordium. Images were obtained in three or four selected views in each patient. The data were stored as a series of $11 \mathrm{R}$-wave triggered intervals between each QRS complex to a total of four million counts recorded in approximately five minutes for each view.

\section{Case reports}

CASE 1

A 10-year-old boy was admitted to Guy's Hospital for dermatological investigation. Since birth he had been heavily freckled and both parents were freckled. At 2 years of age two tumours were excised from his neck with a local recurrence five years later. Histology of all three specimens showed myxosarcoma of low-grade malignancy.

There were no cardiovascular symptoms. Physical examination showed a red-haired boy with extensive freckling. Pulse rate was 76 per minute with sinus arrhythmia. Blood pressure was $110 / 70 \mathrm{mmHg}$. Mitral pansystolic and mid-diastolic murmurs were present intermittently and varied with posture. The remainder of the examination was normal.

Haemoglobin level and leucocyte count were normal. The erythrocyte sedimentation rate was 


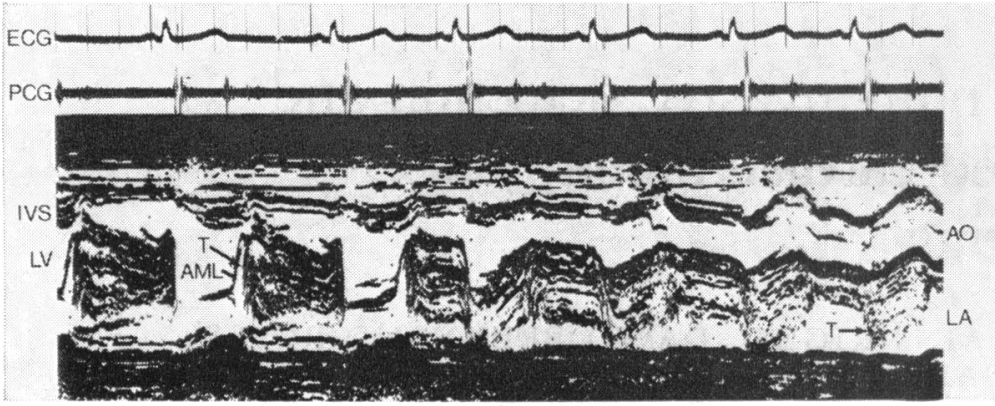

Fig. 1 Echocardiogram and phonocardiogram (PCG) from case 1. In this sweep from left ventricle $(L V)$ to aorta $(A O)$ the myxoma $(T)$ gives rise to multiple echoes between the mitral leaflets in diastole. In systole the tumour appears to occupy the entire depth of the left atrium (LA). IVS, intraventricular septum; $A M L$, anterior mitral leaflet.

raised (43 mm/h, Westergren). The electrocardiogram was normal. The chest radiograph showed left atrial enlargement and prominent upper lobe pulmonary vasculature. An echocardiogram showed a tumour which was seen in the left atrium during systole and appeared between the mitral leaflets in diastole (Fig. 1). The echocardiographic appearance of the pulmonary valve suggested pulmonary hypertension. Gated cardiac blood pool images were acquired in four views. The tumour caused a photondeficient area in the left atrium which moved towards the ventricle in diastole (Fig. 2). Right heart catheterisation confirmed pulmonary hypertension (pulmonary artery pressure $45 / 20 \mathrm{mmHg}$ ). A right ventricular angiogram was normal but follow-through outlined a large left atrial tumour.

At operation a gelatinous tumour occupied the left atrium and bulged through the foramen ovale. A small, separate tumour was attached to the right atrial wall opposite the foramen. Both tumours were removed. Recovery was uneventful. Tumour histology was typical of atrial myxoma. Postoperative echocardiography showed no evidence of residual atrial tumour and normal mitral valve motion.

\section{CASE 2}

A 56-year-old woman was transferred to Guy's Hospital with unstable angina pectoris. Inferior myocardial infarction, confirmed by electrocardiogram and enzyme changes, had occurred four months previously and a probable pulmonary infarct occurred a fortnight before transfer. There was a history of malaise, weight loss, and breathlessness for one year, but no history of rheumatic fever.

On arrival the patient appeared ill. She had a regular pulse of 70 per minute with blood pressure $125 / 85$ mmHg. Pansystolic and mid-diastolic murmurs were present. There were signs of pulmonary hypertension and of a left pleural effusion.

Haemoglobin was $11.9 \mathrm{~g} / \mathrm{dl}$, with a normal leucocyte count and high erythrocyte sedimentation rate $(55 \mathrm{~mm} / \mathrm{h}$-Westergren). The electrocardiogram showed sinus rhythm with widespread $T$ wave flattening or inversion, and $Q$ waves in the inferior leads. The chest radiograph showed a left pleural effusion, cardiomegaly, and pulmonary venous congestion. Echocardiography showed an apparently small $(1.5 \mathrm{~cm}$ depth) left atrial tumour
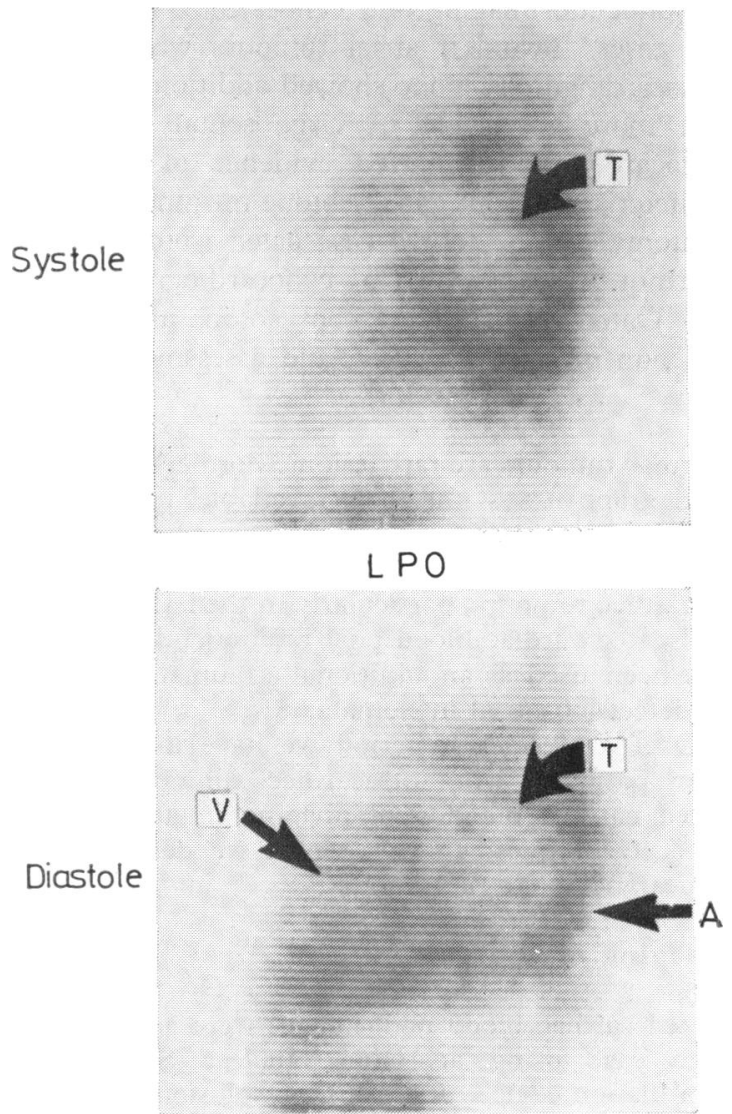

Fig. 2 Systolic and diastolic images from gated cardiac blood pool study in the left posterior oblique (LPO) projection from case 1. During ventricular systole the myxoma $(T)$ is seen as a photon-deficient area high in the left atrium. In diastole the tumour has moved down to the atrioventricular junction and separates tracer activity in the ventricle (V) from that in atrium ( $A)$. 


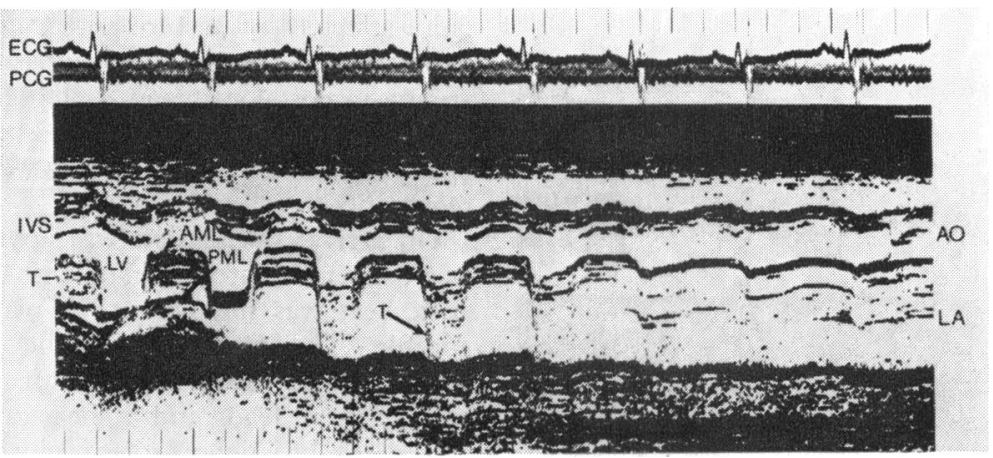

Fig. 3 Echocardiogram and phonocardiogram (PCG) from case 2. A sweep from left ventricle (LV) to aorta (AO) showing multiple echoes immediately behind the anterior mitral leaflet $(A M L)$ in diastole. Echoes from the tumour $(T)$ are also recorded from the left atrium ( $L A)$ during systole. In contrast to Fig. 1 the posterior mitral leaflet (PML) moves anteriorly in diastole, indicating additional mitral valve stenosis.

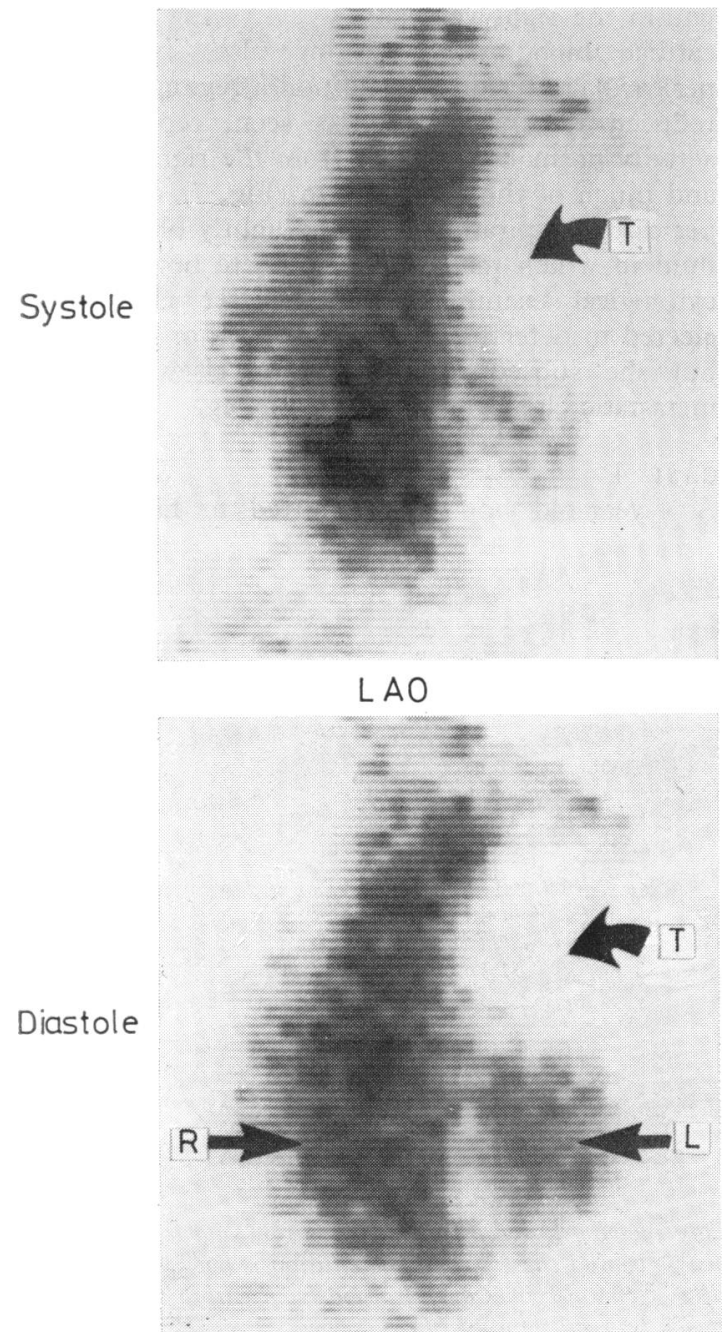

Fig. 4 Systolic and diastolic gated cardiac blood pool images in the left anterior oblique ( $L A O)$ view from case 2. Normal tracer activity is seen in the right ventricle $(R)$ and in the left ventricle $(L)$.

However, no tracer activity is recorded from the region of the left atrium which was filled by tumour $(T)$.

which appeared immediately behind the anterior mitral leaflet in diastole, and moved abruptly into the left atrium in systole. Furthermore, the posterior mitral leaflet echo moved anteriorly in diastole, indicating additional mitral valve stenosis (Fig. 3). The pulmonary valve echogram suggested pulmonary hypertension. Gated cardiac blood pool images showed a large photon-deficient area in the left atrium (Fig. 4). This moved towards the ventricle in diastole. Cardiac catheterisation confirmed pulmonary hypertension $(77 / 35 \mathrm{mmHg}$, and demonstrated a $22 \mathrm{mmHg}$ end-diastolic gradient between simultaneous pulmonary artery wedge and left ventricular pressures. A left ventricular angiogram showed mitral regurgitation which outlined a large left atrial tumour. This was attached to the anterior mitral leaflet and prolapsed into the ventricle in diastole. Coronary arteriography showed a fistula between the right coronary artery and right atrium and several small vessels to the left atrium suggesting a tumour circulation. The coronary arteries were otherwise normal.

At operation a large tumour (diameter $5 \mathrm{~cm}$ ) filled the left atrium and was adherent to the anterior mitral cusp. On removing the tumour the mitral valve appeared thickened and mildly stenosed. The postoperative course was complicated by atrial fibrillation. Tumour histology showed a fibrosarcoma. Repeat echocardiography showed no evidence of residual tumour, but the pattern of mitral stenosis persisted (Fig. 5). 


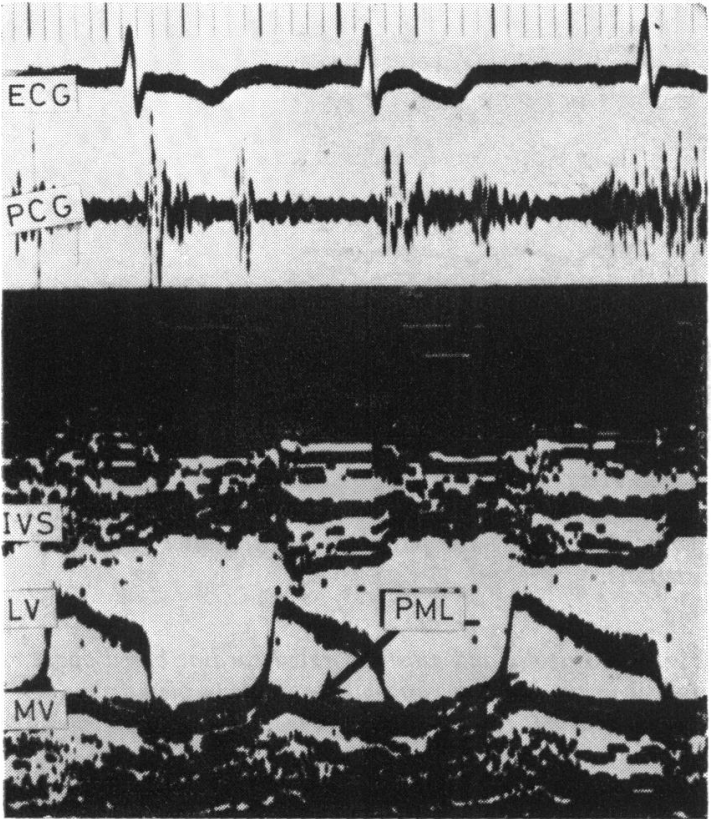

Fig. 5 Postoperative echocardiogram and phonocardiogram (PCG) from case 2 showing persistence of mild mitral stenosis, indicated by slight anterior motion of the posterior mitral leaflet (PML) in early diastole. $M V$, mitral valve.

CASE 3

A 21-year-old Iranian girl was transferred to Guy's Hospital for investigation of suspected pericarditis. She gave a history of increasing sharp praecordial pain, affected by posture and respiration, for three months. Ten years previously a chondrosarcoma, arising in costal cartilage at the left sternal border, had been excised, but until the present illness the patient had remained well, with no sign of recurrence.

On arrival the patient was apyrexial. Pulse rate was 82 per minute, regular, with no paradox. The apex beat was impalpable. A pericardial rub was audible. The remainder of the examination was normal. The electrocardiogram showed reduced voltage in all leads and a chest radiograph showed cardiomegaly. Echocardiography was technically difficult, but confirmed the presence of a large pericardial effusion. Intracardiac structures were poorly defined, and, in particular, definition of the right ventricular/septal endocardium was prevented by the recording of multiple echoes, apparently from within the right ventricular cavity (Fig. 6). Gated cardiac blood pool imaging also showed the pericardial effusion; in addition a region of greatly reduced tracer activity was seen, replacing the activity normally recorded from the right ventricle and much of the right atrium (Fig. 7). Diagnostic pericardial aspiration disclosed lightly bloodstained fluid in which malignant cells were not found on cytological examination. Thereafter the patient elected to defer further investigation or treatment; but she subsequently presented elsewhere with metastatic chondrosarcoma in the leg.

\section{CASE 4}

A 4-year-old boy was admitted to hospital for

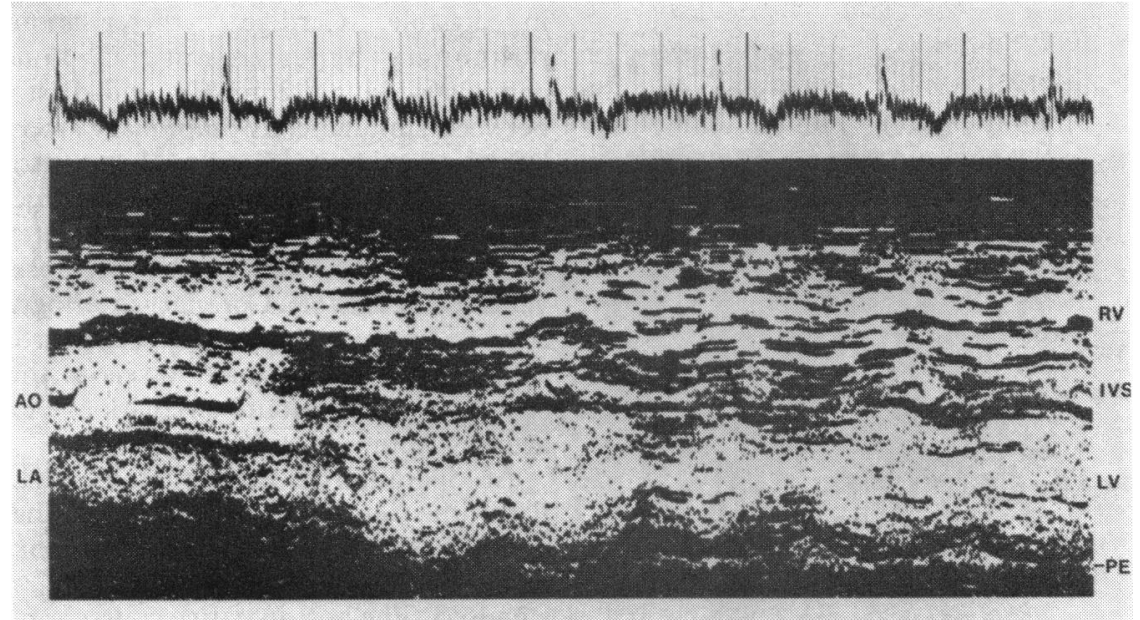

Fig. 6 Echocardiogram from case 3. A technically difficult study showing a sweep from aorta (AO) and left atrium ( $L A$ ) to left ventricle ( $L V)$. A pericardial effusion (PE) is visible behind the $L V$, but the effusion is not well seen anteriorly because of the gain setting for this part of the recording. Several echoes are recorded from within the cavity of the right ventricle ( $R V)$, obscuring the endocardial echo between $R V$ and septum (IVS). 


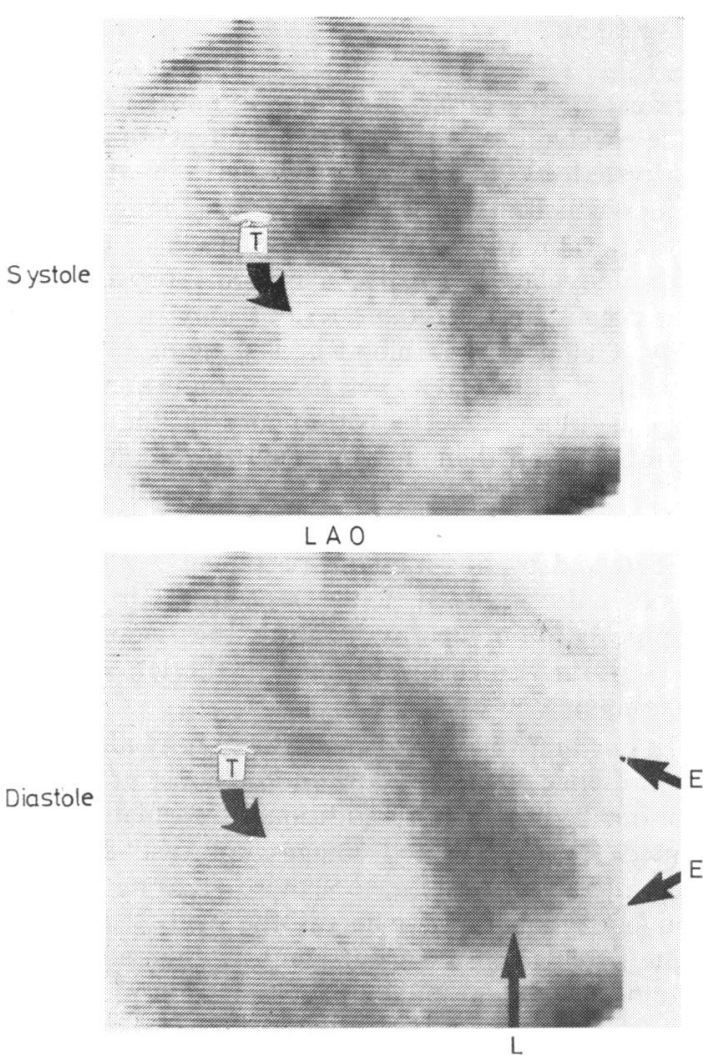

Fig. 7 Systolic and diastolic images in the left anterior oblique (LAO) view from case 3. An area of reduced tracer activity $(T)$ indicates the presence of tumour within the right ventricle, whereas normal tracer activity is recorded from the left ventricle $(L)$. The intracardiac tracer activity is surrounded bv a halo of relatively reduced activity (E) which represents a large pericardial effusion. investigation of a heart murmur, first noted by his family doctor at age 2 years. The patient was easily fatigued but his growth and milestone achievement had been normal. There was no other previous medical history and no family history of heart disease.

On examination, height and weight were at the 70th and 75th centiles for age, respectively. The pulse was regular at 92 per minute and of normal character. Blood pressure was $105 / 60 \mathrm{mmHg}$. The heart was enlarged, with a sustained left parasternal heave and a basal ejection systolic murmur. The remainder of the examination was normal.

Haematological and biochemical screens were normal. The electrocardiogram showed sinus rhythm with a QRS axis of $-120^{\circ}$, complete right bundle-branch block, and pronounced right ventricular hypertrophy. The chest radiograph showed cardiac enlargement. An echocardiogram showed a greatly thickened interventricular septum with reduced motion. Motion of the posterior wall was increased. There was systolic anterior motion of the mitral valve, suggesting left ventricular outflow obstruction but the aortic valve appeared normal (Fig. 8). Pronounced septal hypertrophy was also seen on the gated cardiac blood pool images which also showed increased thickness of the free wall and apex of the left ventricle (Fig. 9). Cardiac catheterisation demonstrated a systolic pressure gradient of $20 \mathrm{mmHg}$ across the left ventricular outflow tract. Intracardiac pressures were otherwise normal. A right ventricular angiogram showed a large mass protruding from the septum into the right ventricle; left ventriculography showed a septal mass encroaching on the left ventricular outflow tract, and hypertrophied papillary muscles.

At operation a large purple mass occupied the upper septum and appeared to extend into the free

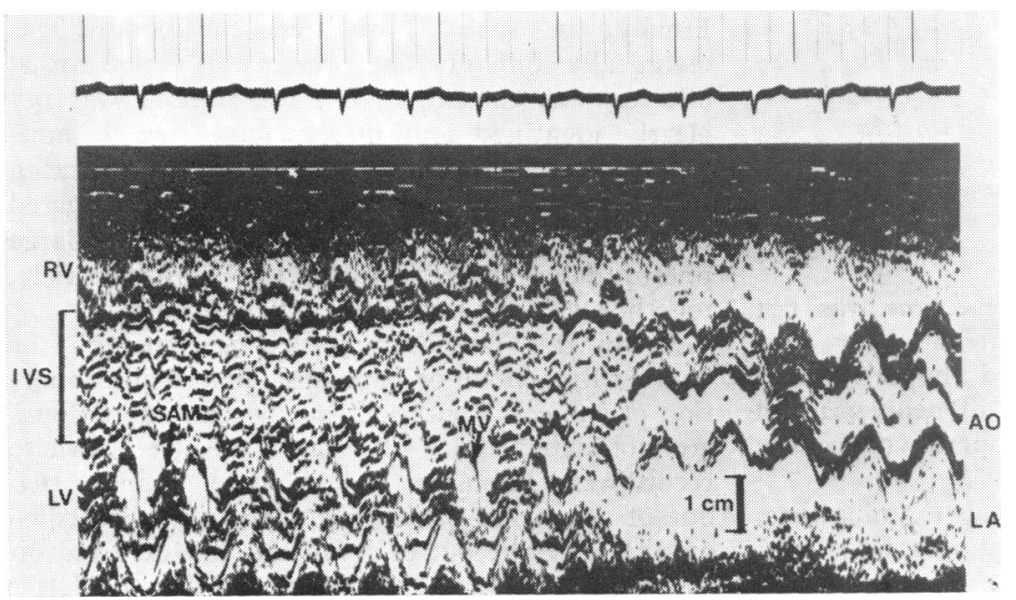

Fig. 8 Echocardiogram from case 4; sweep from the left ventricle ( $L V)$ to aorta $(A O)$ and left atrium (LA). The septum (IVS) is greatly thickened and is hypokinetic. The mitral valve (MV) shows systolic anterior motion ( $S A M$ ) indicating $L V$ outflow obstruction. 


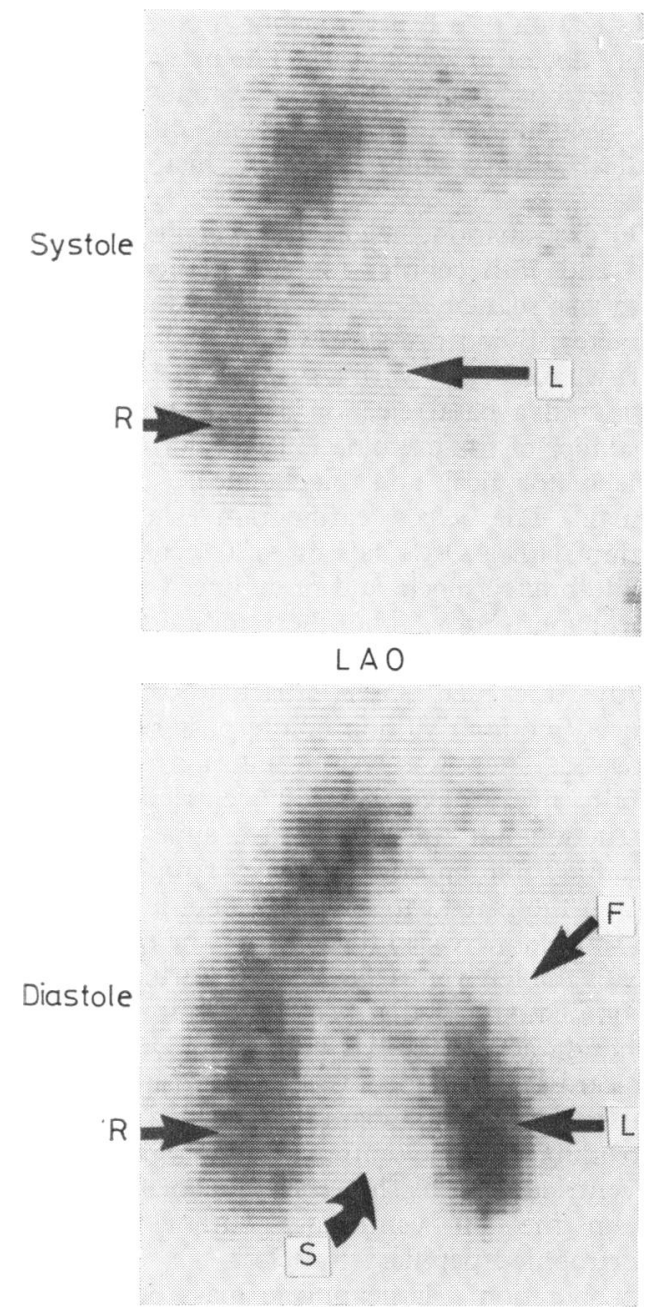

Fig. 9 Systolic and diastolic left anterior oblique gated images from case 4. In diastole normal tracer activity is seen in the right ventricle $(R)$ and left ventricle $(L)$, separated by a wide band of low activity indicating $a$ thickened septum (S). A halo of relatively low tracer activity around the remainder of the left ventricle suggests hypertrophy of the free wall (F). In systole, left ventricular tracer activity is almost obliterated.

wall of the left ventricle. As resection was not feasible a biopsy was taken and the heart was closed again. Histology showed hypertrophied cardiac muscle with no evidence of neoplasia and no myocardial fibre disarray. Postoperative recovery was uneventful.

Both parents have had clinical examinations, electrocardiograms, and echocardiograms, all of which were normal.

\section{Discussion}

Because of the rarity of cardiac tumours and their wide spectrum of non-specific manifestations, ${ }^{5}$ well illustrated by these four cases, atraumatic techniques which allow detection of these lesions have an important place in the investigation of patients with unexplained cardiac symptoms or signs, so that potentially resectable lesions may be detected early. Gated cardiac blood pool imaging is such a technique. Intracavity cardiac tumours may be seen as filling defects within any of the cardiac chambers, and their motion may be demonstrated, as in our first three cases. Thickening of the myocardium (by tumour or hypertrophy) may also be detected by this method, particularly when this involves the interventricular septum, as in hypertrophic cardiomyopathy. ${ }^{6}$ Hence the widening of the septum was easily detected in the left anterior oblique view in case 4 .

In our first two cases echocardiography identified the presence of the tumours and demonstrated their motion. In case 1 little additional information was gained from the gated images; in case 2 gated radionuclide imaging gave a more accurate indication of tumour size, whereas the echocardiogram alone detected mitral valve stenosis. The ability to obtain two-dimensional images in multiple views in case 4 allowed the extent of left ventricular hypertrophy to be defined much more precisely than by single-beam echocardiography. The echocardiogram, on the other hand, detected evidence of left ventricular outflow obstruction which was otherwise demonstrated only by cardiac catheterisation. Neither echocardiography nor gated isotope imaging provided a firm diagnosis in this case, and though the angiogram suggested a septal tumour, operative biopsy also failed to distinguish neoplasm from hypertrophic cardiomyopathy. Finally, in case 3, the echocardiogram was technically difficult. The presence of a pericardial effusion was confirmed, but the tumour was not clearly identified and merely gave rise to nonspecific echoes within the right ventricle, obscuring the septal endocardium. In contrast, the gated cardiac blood pool images clearly showed a large photon-deficient area within the right ventricle, together with the large pericardial effusion.

As in this last example, echocardiography is technically difficult or impossible in a small proportion of subjects, ${ }^{7}$ and occasionally cardiac tumours are not detected by ultrasound despite adequate recordings. ${ }^{8}$ Pohost et al. ${ }^{4}$ failed to diagnose two out of seven left atrial myxomata by echocardiography, and one out of seven was not detected on gated images. These authors suggest that this latter 
failure was the result of multiple lobulation of the myxoma, allowing the passage of blood between the lobules and resulting in the recording of tracer activity from the region occupied by the tumour. It is just this type of tumour in which multiple surfaces might be expected to give rise to multiple ultrasonic echoes, which allow easy echocardiographic diagnosis. Conversely, a solid tumour of uniform density may be less easy to detect by echocardiography and more readily identified by isotope imaging.

Thus gated cardiac blood pool radionuclide imaging appears to be complementary to echocardiography for the atraumatic detection and assessment of cardiac tumours. It appears to be of particular diagnostic value in patients in whom echocardiography is technically difficult or nondiagnostic. The demonstration of a cardiac tumour by either of these techniques may permit surgical exploration without the need for cardiac catheterisation and contrast angiography.

\section{References}

1 Schattenberg TT. Echocardiographic diagnosis of left atrial myxoma. Mayo Clin Proc 1968; 61 : 620-7.

2 Wolfe SB, Popp RL, Feigenbaum H. Diagnosis of atrial tumors by ultrasound. Circulation 1969; 39: 615-22.

3 Bonte FJ, Curry TS. Technetium-99m HSA blood pool scan in diagnosis of an intracardiac myxoma. f Nucl Med 1969; 8: 35-9.

4 Pohost GM, Pastore JO, McKusick KA, et al. Detection of left atrial myxoma by gated radionuclide cardiac imaging. Circulation 1977; 55: 88-92.

5 Selzer A, Sakai FJ, Popper RW. Protean clinical manifestations of primary tumors of the heart. $A m \mathcal{F}$ Med 1972; 52: 9-18.

6 Pohost GM, Vignola PA, McKusick KA, et al. Hypertrophic cardiomyopathy. Evaluation by gated cardiac blood pool scanning. Circulation 1977; 55: 92-9.

7 Gramiak R. Echocardiography in acquired heart disease. In: King DL, ed. Diagnostic ultrasound. St Louis: CV Mosby, 1974: 84.

8 Guiliani ER, Lemire F, Schattenberg TT. Unusual echocardiographic findings in a patient with left atrial myxoma. Mayo Clin Proc 1978; 53: 469-72.

Requests for reprints to Dr David Pitcher, Department of Cardiology, Guy's Hospital, London SE1 9RT. 